\title{
Intraoperative Surgical Wound Contamination May Not Lead to Surgical-Site Infection in Patients Undergoing Clean Orthopaedic Procedures
}

\author{
John Ashutosh Santoshi ${ }^{1}$ Prateek Behera ${ }^{1}$ Ayush Gupta ${ }^{2}$ Archa Sharma ${ }^{2}$ Virendra Kumar Verma ${ }^{1}$ \\ Udit Agrawal $^{1}$ Shashank Purwar ${ }^{2}$ \\ ${ }^{1}$ Department of Orthopaedics, All India Institute of Medical Sciences, \\ Bhopal, Madhya Pradesh, India \\ ${ }^{2}$ Department of Microbiology, All India Institute of Medical Sciences, \\ Bhopal, Madhya Pradesh, India \\ Address for correspondence Shashank Purwar, MD, PhD, Department \\ of Microbiology, All India Institute of Medical Sciences, Bhopal, \\ Madhya Pradesh, 462020, India \\ (e-mail: shashank.microbiology@aiimsbhopal.edu.in).
}

J Lab Physicians 2022;14:284-289.

\begin{abstract}
Keywords

- Clean orthopaedic surgery

- intraoperative contamination

- surgical-site infection

- perioperative

- aerobic culture

- SSI rate

Objectives Surgical-site infections (SSIs) can complicate virtually any surgical procedure. While SSI can result from numerous causes, contamination of the surgical field can also contribute to it. Intraoperative bacterial contamination during clean orthopaedic procedures can be detected using perioperative cultures. We hypothesized that perioperative cultures could be used to predict possibility of development of SSI in patients undergoing clean orthopaedic surgeries.

Materials and Methods We conducted a prospective cohort study at a tertiary care hospital over a 2-year period. Intraoperative surgical wound lavage fluid and closed suction drain tip obtained in the postoperative period were sent for aerobic culture. All patients were followed up to look for the development of SSI for a period of at least 30 days for those undergoing nonimplant surgery, and 90 days for those with implant surgery.

Statistical Analysis Means with standard deviation of the continuous data were calculated. Fisher's exact test and chi-square test were used for the analysis of the categorical variables. Relative risk and odds ratio were calculated to evaluate the association of the parameters under study with SSI.

Results A total of 384 patients satisfying the inclusion and exclusion criteria were included. Perioperative cultures detected surgical wound contamination in 39 patients (10.1\%). Forty-five patients (11.7\%) developed SSI during the follow-up period. Skin commensals constituted $59 \%$ of perioperative contaminants and accounted for $20 \%$ of
\end{abstract}

This research was carried out at the All India Institute of Medical Sciences, Bhopal, Madhya Pradesh, India.

published online February 9, 2022
DOI https://doi.org/ $10.1055 / \mathrm{s}-0042-1742422$ ISSN $0974-2727$. (c) 2022. The Indian Association of Laboratory Physicians. All rights reserved.

This is an open access article published by Thieme under the terms of the Creative Commons Attribution-NonDerivative-NonCommercial-License, permitting copying and reproduction so long as the original work is given appropriate credit. Contents may not be used for commercial purposes, or adapted, remixed, transformed or built upon. (https://creativecommons.org/ licenses/by-nc-nd/4.0/)

Thieme Medical and Scientific Publishers Pvt. Ltd., A-12, 2nd Floor, Sector 2, Noida-201301 UP, India 
the SSIs. The relative risk of developing SSI with perioperative contamination was 0.41 (95\% confidence interval: 0.09-1.63).

Conclusion Intraoperative surgical-site contaminants could be detected using perioperative cultures. However, these contaminants did not lead to SSI. Timely treatment of perioperative contamination with appropriate antibiotics and local wound care probably helped in the reduction of SSI.

\section{Introduction}

Surgical-site infections (SSIs) are wound infections occurring after an operative procedure. ${ }^{1}$ They can complicate virtually any surgical procedure and carry high morbidity and mortality. ${ }^{1-4}$ While most of the SSIs are multifactorial events, they are thought to originate from intraoperative surgical wound contamination. ${ }^{2,5}$ While it is assumed that all surgical wounds get contaminated to some degree, ${ }^{6}$ intraoperative contamination rates of 23 to $63 \%$ have been reported in clean orthopaedic surgery. ${ }^{7}$

Surgical wound lavage, usually undertaken prior to wound closure, is reported to reduce the bacterial load at the surgical site through removal of dead or damaged tissue, metabolic waste, and wound exudate on which the microorganisms can thrive, and thus, reduce the likelihood of the SSI. ${ }^{1,8}$ While surgical wound lavage is performed in most orthopaedic procedures, there are no guidelines with regard to its timing. Intraoperative contamination can be detected using lavage fluid culture. ${ }^{9}$

Use of a closed suction drain in the postoperative period is another common tool used in many orthopaedic surgeries. $^{2,10-12}$ The underlying concept of using it is to prevent haematoma formation, thereby promoting uneventful wound healing. It has been suggested that a positive drain tip culture may help in predicting SSI while a negative culture would virtually exclude the possibility of SSI. ${ }^{2}$

It may be possible to indirectly detect intraoperative bacterial contamination by using surgical wound lavage fluid and postoperative drain tip culture as surrogates. Based on this background knowledge, we hypothesized that in clean orthopaedic surgeries, intraoperative surgical-site contamination may lead to subsequent SSI, and if this is true, then, perioperative cultures would help detect surgical-site contamination at an early stage paving the way for initiating appropriate antimicrobial therapy.

\section{Materials and Methods}

We conducted a prospective cohort study in a tertiary care teaching hospital over a 2-year period from April 2017 to March 2019 after obtaining approval from the institutional human ethics committee (reference no. IHEC-LOP/2015/ IM0074). Patients undergoing clean elective orthopaedic surgical procedures during the study period were included after obtaining consent. The surgical wounds were grade I/ "clean" as per the Centers for Disease Control and Prevention
(CDC) surgical wound classification system. ${ }^{13}$ Perioperative surgical wound culture samples, viz., intraoperative surgical wound lavage fluid and closed suction drain tip obtained in the postoperative period, were sent for aerobic cultures to our microbiology laboratory. The exclusion criteria were patients with history of prior infection at the surgical site, patients with total leucocyte count less than 4,000/cu mm, and patients with medical conditions which are likely to be treated with antibiotics during the study period, like concomitant urinary tract infection, chest infection, etc. Additionally, patients undergoing day-care surgery, procedures of short surgical duration where intraoperative lavage was not performed, those for whom drains were not placed, and patients with open wounds were also excluded.

All patients received intravenous cefuroxime as surgical antibiotic prophylaxis (SAP) within 1 hour of surgical incision, which was continued for 48 hours after the surgery as per our departmental protocol. Skin preparation protocol for the surgical site included the use of povidone iodine 7.5\% scrub solution for prewash and $10 \%$ solution for paint. Intraoperative surgical wound lavage fluid (3-5 mL) was collected just before closure of the wound and sent for aerobic culture. The closed suction drain tip, which was removed at 24 to 48 hours postoperatively, was also sent for aerobic culture.

The wound lavage fluid was inoculated in the blood culture bottles and incubated in automated blood culture system (BacT-Alert 3D 480, BioMurieux, Durham, North Carolina, United States) for up to 7 days or till the time growth was indicated, whichever was earlier. Subsequently, the broth from positive blood culture bottles was sub-cultured on blood agar (enriched medium) and MacConkey agar (differential media). The drain tips were rolled on the blood culture plates. More than 10 colonies of same organisms or lesser colonies in case of known pus forming bacteria like Staphylococcus aureus were considered significant for further identification. The bacterial isolates were identified by conventional microbiological methods and the VITEK 2 compact system (BioMurieux, Durham, North Carolina, United States). Antimicrobial susceptibility testing was done by the disc diffusion method, according to the Clinical \& Laboratory Standards Institute guidelines and the VITEK 2 system. Coagulase-negative Staphylococcus (CoNS), Corynebacterium species, Bacillus species (not anthrasis), Micrococcus species, Streptococcus viridans ( $\alpha$ hemolytic streptococci), and Propionibacterium acnes were reported as skin contaminants. ${ }^{14}$

In case any perioperative sample (lavage fluid and/or drain tip) showed bacterial growth, the patient was treated 
Table 1 Details of site and type of surgery, intraoperative contamination, and SSI

\begin{tabular}{|l|l|l|l|l|l|}
\hline \multirow{2}{*}{$\begin{array}{l}\text { Anatomical location } \\
\text { of the surgical } \\
\text { procedure }\end{array}$} & \multirow{2}{*}{ Number of patients } & \multicolumn{2}{|l|}{ Type of surgery } & $\begin{array}{l}\text { Intraoperative } \\
\text { contamination }\end{array}$ & $\begin{array}{l}\text { SSI (whether any } \\
\text { patient had intraoperative } \\
\text { contamination) }\end{array}$ \\
\cline { 3 - 6 } & & Implant & Nonimplant & & $2(0)$ \\
\hline Shoulder & 14 & 10 & 4 & 0 & $1(0)$ \\
\hline Arm & 31 & 27 & 4 & 4 & $1(0)$ \\
\hline Elbow & 15 & 12 & 3 & 3 & 0 \\
\hline Forearm & 30 & 22 & 8 & 0 & $2(0)$ \\
\hline Hand and wrist & 48 & 39 & 9 & 3 & 0 \\
\hline Spine & 1 & 1 & 0 & 0 & $6(0)$ \\
\hline Hip & 68 & 60 & 8 & 5 & $14(0)$ \\
\hline Femur & 76 & 73 & 3 & 10 & $2(0)$ \\
\hline Knee & 15 & 13 & 2 & 3 & $14(1)^{\mathrm{a}}$ \\
\hline Leg & 64 & 54 & 10 & 9 & $3(1)^{\mathrm{b}}$ \\
\hline Foot and ankle & 22 & 20 & 2 & 2 & $45(2)$ \\
\hline Total & 384 & 332 & 53 & 39 & \\
\hline
\end{tabular}

Abbreviation: SSI, surgical site infection.

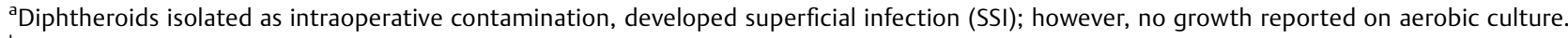

${ }^{\mathrm{b}}$ Citrobacter isolated as intraoperative contamination; subsequently, had SSI with E. coli.

with appropriate antibiotics and local wound care, as indicated. All patients were followed up at regular intervals (postdischarge from the hospital), as deemed appropriate for the procedure, for a period of at least 30 days for those undergoing nonimplant surgery, and 90 days for any implant surgery. In case of suspicion of SSI, blood parameters like Creactive protein, erythrocyte sedimentation rate, and total leucocyte count were performed, and any surgical-site discharge was collected taking care to avoid contamination and sent for aerobic culture. The report from this sample was correlated with the perioperative culture reports. SSIs were classified as superficial or deep according to the CDC criteria $^{15}$ and were treated according to standard protocols.

\section{Statistical Analysis}

The data were tabulated and analyzed using SPSS version 20 (IBM Corp, Armonk, New York, United States). Means with standard deviation of the continuous data were calculated. Fisher's exact test and chi-square test were used for analysis of the categorical variables. Further, for the purpose of analysis, we broadly grouped the surgeries based on anatomical location. Relative risk and odds ratio were calculated to evaluate the association of the parameters under study with SSI. A p-value less than 0.05 was taken to indicate statistical significance.

\section{Results}

Out of the 384 patients included in the study, implant surgery was performed for 331 (86\%) patients. The number of females was 114 (29.6\%). The mean age of the patients was $37.05 \pm 19.24$ years. The details of anatomical location of the surgery are given in - Table $\mathbf{1}$. The intraoperative surgical wound lavage fluid culture was sterile in 357 patients while the drain tip culture was sterile in 369 patients. For three patients, both the perioperative samples were positive and grew Enterobacter, methicillin-sensitive $S$. aureus, and methicillin-resistant CoNS, respectively; however, none of them developed SSI. - Table 2 shows the details of intraoperative contaminants detected on aerobic culture.

Forty-five patients developed SSI during the follow-up period; three superficial and the remaining deep. All except three had undergone implant surgery. The overall SSI rate was $11.7 \%$; it was $12.6 \%$ for patients with implant surgery and $5.6 \%$ for those with nonimplant surgery. No organism could be identified in four of these patients. Only 2 patients out of 39 who had perioperative surgical-site contamination developed SSI. One of these patients had a soft-tissue surgery of the leg. He grew diphtheroids in the perioperative culture, had superficial SSI, but the culture from the surgical site was negative. The second patient had an implant surgery in the foot and grew Citrobacter freundii perioperatively. He developed deep SSI and grew Escherichia coli. - Table 3 shows the microorganisms isolated in the SSIs.

The relative risk of developing SSI in patients who had a positive perioperative culture was 0.41 (95\% confidence interval: 0.09-1.63). Using Fisher's exact test, no significant association of a patient's gender with the type of surgery $(p=0.105)$, perioperative lavage culture and drain tip culture being sterile ( $p=0.274$ and 0.776 ), and presence or absence of SSI ( $p=0.081$ ) was found. However, there was a statistically significant difference in the number of SSIs between the lower limb and upper limb surgeries $(p=0.000)$.

\section{Discussion}

Intraoperative surgical wound lavage fluid and postoperative suction drain tip can be considered as surrogate indicators of intraoperative contamination as they provide direct samples 
Table 2 Details of intraoperative contaminants isolated in comparison to previous studies

\begin{tabular}{|c|c|c|c|c|c|c|c|}
\hline Bacteria & $\begin{array}{l}\text { Davis et } \mathrm{al}^{5} \\
(n=100)\end{array}$ & $\begin{array}{l}\text { Byrne et al }{ }^{17} \\
(n=80)\end{array}$ & $\begin{array}{l}\text { Al-Maiyah } \\
\text { et al } \\
(n=50)\end{array}$ & $\begin{array}{l}\text { Beldame } \\
\text { et al }{ }^{16} \\
(n=29)\end{array}$ & $\begin{array}{l}\text { Jonsson et } \mathrm{al}^{7} \\
(n=90)\end{array}$ & $\begin{array}{l}\text { Ahn et al }{ }^{18} \\
(n=133)\end{array}$ & $\begin{array}{l}\text { Present study } \\
(n=384)\end{array}$ \\
\hline MSSA & $1(1)$ & - & 7 & 0 & 9 & $5(2)$ & 3 \\
\hline MRSA & - & - & - & - & - & - & 1 \\
\hline Escherichia coli & - & - & - & - & - & - & 3 \\
\hline Klebsiella spp. & - & - & - & - & - & - & 2 \\
\hline Enterobacter spp. & - & - & - & - & - & - & 2 \\
\hline Other GNB ${ }^{\mathrm{a}}$ & 4 & 2 & 3 & 0 & 1 & 3 & $6(1)$ \\
\hline Diphtheroids & 2 & - & 10 & 1 & 1 & - & $1(1)$ \\
\hline CoNS & 86 & - & 73 & 15 & $26(1)$ & $39(5)$ & 4 \\
\hline MRCoNS & - & - & - & 3 & - & - & 8 \\
\hline $\begin{array}{l}\text { Other skin } \\
\text { commensals }\end{array}$ & - & 15 & - & - & 1 & - & 10 \\
\hline Polymicrobial & - & 3 & - & - & 2 & 1 & $2^{b}$ \\
\hline Others $^{c}$ & 11 & 9 & 13 & 4 & 1 & $1(1)$ & 0 \\
\hline
\end{tabular}

Abbreviations: CoNS, coagulase-negative Staphylococcus; GNB, gram-negative bacteria; MRCoNS, methicillin-resistant CoNS; MRSA, methicillinresistant Staphylococcus aureus; MSSA, methicillin-sensitive Staphylococcus aureus.

${ }^{a}$ Other GNB included Proteus mirabilis, Pseudomonas spp., Serratia marcescens, Citrobacter spp., Acinetobacter spp.

${ }^{\mathrm{b}}$ Polymicrobial included Proteus mirabilis + MSSA.

'Others included Streptococcus spp., Micrococcus spp., Bacillus spp., Micrococcus spp.; SSI shown in parentheses, if contamination was detected perioperatively.

Table 3 Microorganisms isolated in SSIs in comparison to previous studies

\begin{tabular}{|c|c|c|c|c|c|c|}
\hline Bacteria & $\begin{array}{l}\text { Al-Mulhim et } \mathrm{al}^{3} \\
(n=3,096)\end{array}$ & $\begin{array}{l}\text { Rajkumari et } \mathrm{al}^{25} \\
(n=852)\end{array}$ & $\begin{array}{l}\text { Ahn et al }{ }^{18} \\
(n=133)\end{array}$ & $\begin{array}{l}\text { Khan et al } 26 \\
(n=103)\end{array}$ & $\begin{array}{l}\text { Maksimović } \\
\text { et } \mathrm{al}^{27} \\
(n=277)\end{array}$ & $\begin{array}{l}\text { Present study } \\
(n=384)\end{array}$ \\
\hline MSSA & \multirow{2}{*}{$\begin{array}{l}23 \\
(\mathrm{MSSA}+\mathrm{MRSA})\end{array}$} & - & 2 & 3 & 5 & 6 \\
\hline MRSA & & 8 & - & - & 19 & 8 \\
\hline Klebsiella spp. & 3 & 8 & - & 1 & 10 & 7 \\
\hline Escherichia coli & 3 & 6 & - & 1 & - & 5 \\
\hline Pseudomonas spp. & 15 & 8 & - & - & 9 & 4 \\
\hline Enterobacter spp. & 14 & 1 & - & - & - & 3 \\
\hline Acinetobacter spp. & 17 & 10 & - & - & 20 & 3 \\
\hline CoNS & - & - & 5 & - & 4 & 2 \\
\hline Other skin commensals & - & 2 & - & - & - & 6 \\
\hline Polymicrobial & - & - & - & - & 53 & $1^{\mathrm{a}}$ \\
\hline Others & 7 & 3 & 1 & 1 & 16 & \\
\hline
\end{tabular}

Abbreviations: CoNS, coagulase-negative Staphylococcus; MRSA, methicillin-resistant Staphylococcus aureus; MSSA, methicillin-sensitive Staphylococcus aureus.

${ }^{\mathrm{a} C o N S}+$ Acinetobacter baumannii.

from the surgical site itself. Host defense is the primary determinant of whether intraoperative contamination will get established as a clinical infection and the first few hours are most critical in this regard. ${ }^{6}$ We believe that our method of obtaining two separate samples, at least 24 to 48 hours apart, from the surgical site ensured that this important factor was taken care of.
It is assumed that all surgical wounds become contaminated to some degree, ${ }^{6}$ and surgical wound contamination rates of 23 to $63 \%$ have been reported in the literature ${ }^{5,16-19}$; this was $10.1 \%(39 / 384$ ) in the present study (-Table 2 ). The probable reason for the low intraoperative contamination rate in the present study could be due to surgical wound lavage which has been reported to wash away the contaminants, and dilute and 
reduce the size of inoculum. This could also be attributed to the differences in sampling techniques.

The overall SSI rate in the present study was $11.7 \%$, which is considered high for clean orthopaedic surgeries. The SSI rates in such surgeries have been reported from $1 \%^{17}$ to $6.2 \%^{13}$ in the developed countries, and up to $15 \%^{20}$ in developing countries. The rate of SSI for the lower extremity was $16 \%$ (37/231), which accounted for $82 \%$ of all cases with SSIs; a similar trend of higher SSI rate in the lower extremity has been noted in other studies as well. ${ }^{13,21}$ Moreover, most of the patients in the present study underwent implant surgery, which is known to have higher rates of SSI, and are difficult to evaluate without the use of post-discharge surveillance. ${ }^{1,20,22}$ We ensured adequate post-discharge surveillance by following up all patients with implant surgery for at least 90 days as per the $\mathrm{CDC}$ guidelines. ${ }^{15}$

Although intraoperative contamination was common in the present study, first, it did not correlate with development of SSI; thus, positive perioperative cultures were not a reliable predictor of SSI. Only 2 patients out of the 39 who had intraoperative contamination developed SSI-a rate of $5 \%$. This could be attributed to the fact that all patients with perioperative culture positivity were treated with appropriate antibiotics and wound care. Second, SSIs are a complex interplay of host defenses and virulence of the infecting microorganism $^{7}$; and we studied only one factor, that is, the presence of perioperative contamination.

The bacteriology of the contaminants as well as SSI in this study is worrisome. The standard SAP in our setting has been first- or second-generation cephalosporin which would not cover $25.6 \%$ of the contaminants, which were methicillinresistant. ${ }^{23}$ Similar findings have been reported by other authors. ${ }^{16}$ Furthermore, gram-negative bacteria constituted $38.5 \%$ of the perioperative contaminants and caused more than $50 \%$ of the SSIs; these were not covered by the routine SAP. Revisiting our perioperative protocols in view of the antibiogram is warranted.

Skin preparation is another concern as skin commensals constituted $59 \%$ of intraoperative contaminants, and accounted for $20 \%$ of SSIs. This could also reflect poor operating room cleaning and sanitization protocols as bacterial load on the surface of operating table or the supports used during surgery are reported to lead to cross-infection and SSI. ${ }^{4,24}$ The microorganisms causing SSI in this study are comparable to reports by other authors (- Table 3). ${ }^{3,18,25-27}$ Al-Mulhim et al, ${ }^{3}$ Rajkumari et al, ${ }^{25}$ and Maksimović et al $^{27}$ reported on the prevalence of SSI in orthopaedic practice, which included closed as well as open cases, whereas Ahn et $\mathrm{al}^{18}$ and Khan et $\mathrm{al}^{26}$ reported on SSIs in clean orthopaedic cases only.

To the best of our knowledge, no previous study has evaluated intraoperative surgical wound contamination using perioperative samples. Although using the current methodology we were able to detect surgical-site contamination, we were not able to predict the occurrence of SSI using that information. While surgical-site contamination was quite common, we still lack the understanding of exactly when the surgical site starts to develop infection.

\section{Limitations}

First, we recognize that most SSIs are multifactorial events. However, we focused on intraoperative surgical wound contamination only, assuming this to be the most significant factor causing SSI. Other factors like experience and skill of the operating surgeon, soft-tissue handling, surgery duration, body mass index, comorbidities, concurrent tobacco use, and perioperative allogenic blood transfusion are important and have been implicated in SSI. Second, we did not perform anaerobic culture which could have detected a higher level of contamination. However, anaerobes are not routinely reported to cause SSI in clean orthopaedic procedures. $^{28}$ Another limitation of our study is the limited number of patients with intraoperative contamination. Out of consecutive 384 patients approached, we could detect surgical-site contamination in 39 patients. Fewer numbers of patients with intraoperative contamination render a lower power to identify difference in incidence of SSIs in intraoperatively contaminated versus noncontaminated groups. Therefore, the effect size observed in the study cannot be generalized. We believe that a future study with participation of multiple centers with larger samples is required to further assess this hypothesis of predicting the development of SSI using the perioperative cultures. We also recognize the need to explore gaps in our protocols as well as antiseptic routines and operating room behavioral practices to improve patient outcomes.

\section{Conclusion}

With the above-mentioned limitations, the results of our study suggest that intraoperative contamination may not result in SSI. Use of perioperative cultures in clean orthopaedic surgeries helped in detecting intraoperative surgical-site contamination, which was common, but did not correlate with the occurrence of SSI. Timely treatment of perioperative contamination with appropriate antibiotics and local wound care probably helped in the reduction of SSI.

\section{Authors' Contribution}

This submission represents honest and significant work from all authors. J.A.S. and S.P. conceived the idea for the study and designed the protocol. J.A.S., V.K.V., and P.B. performed the surgeries. S.P., A.G., and A.S. performed the microbiological laboratory tests. A.S. and U.A. collected the data. J.A.S., S.P., P.B., V.K.V., and A.S. were involved in the analysis and interpretation of the results. J.A.S. and P.B. wrote the first draft. All authors were involved in the editing of the manuscript and gave approval to the final draft.

\section{Ethical Approval}

Approval for the study was provided by the Institutional Human Ethics Committee of All India Institute of Medical Sciences, Bhopal, India (Reference no. IHEC-LOP/2015/ IM0074).

\section{Conflict of Interest}

None declared. 


\section{References}

1 Norman G, Atkinson RA, Smith TA, et al. Intracavity lavage and wound irrigation for prevention of surgical site infection. Cochrane Database Syst Rev 2017;10(10):CD012234

2 Sankar B, Ray P, Rai J. Suction drain tip culture in orthopaedic surgery: a prospective study of 214 clean operations. Int Orthop 2004;28(05):311-314

3 Al-Mulhim FA, Baragbah MA, Sadat-Ali M, Alomran AS, Azam MQ. Prevalence of surgical site infection in orthopedic surgery: a 5year analysis. Int Surg 2014;99(03):264-268

4 Dallolio L, Raggi A, Sanna T, et al. Surveillance of environmental and procedural measures of infection control in the operating theatre setting. Int J Environ Res Public Health 2017;15(01):46

5 Davis N, Curry A, Gambhir AK, et al. Intraoperative bacterial contamination in operations for joint replacement. J Bone Joint Surg Br 1999;81(05):886-889

6 Belda FJ, Aguilera L, García de la Asunción J, et al; Spanish Reduccion de la Tasa de Infeccion Quirurgica Group. Supplemental perioperative oxygen and the risk of surgical wound infection: a randomized controlled trial. JAMA 2005;294(16):2035-2042

7 Jonsson EÖ, Johannesdottir H, Robertsson O, Mogensen B. Bacterial contamination of the wound during primary total hip and knee replacement. Median 13 years of follow-up of 90 replacements. Acta Orthop 2014;85(02):159-164

8 World Health Organization. Global guidelines for the prevention of surgical site infection. GenevaWorld Health Organization2018. Accessed December 23, 2021: https://www.ncbi.nlm.nih.gov/books/nbk536404/

9 Barnes S, Spencer M, Graham D, Johnson HB. Surgical wound irrigation: a call for evidence-based standardization of practice. Am J Infect Control 2014;42(05):525-529

10 Parker MJ, Livingstone V, Clifton R, McKee A. Closed suction surgical wound drainage after orthopaedic surgery. Cochrane Database Syst Rev 2007;2007(03):CD001825

11 Chandratreya A, Giannikas K, Livesley P. To drain or not drain: literature versus practice. J R Coll Surg Edinb 1998;43(06): 404-406

12 Oguzie GC, Albright P, Ali SH, et al. Prophylactic surgical drainage is associated with increased infection following intramedullary nailing of diaphyseal long bone fractures: a prospective cohort study in Nigeria. SICOT J 2020;6:7

13 Onyekwelu I, Yakkanti R, Protzer L, Pinkston CM, Tucker C, Seligson D. Surgical wound classification and surgical site infections in the orthopaedic patient. J Am Acad Orthop Surg Glob Res Rev 2017;1(03):e022

14 Fairfax MR, Salimnia HBeware of unusual organisms masquerading as skin contaminants. Sepsis - An Ongoing and Significant Challenge. IntechOpen 2012:275-286 [cited 2021 May 18]. Accessed December 23, 2021: https://www.intechopen.com/books/sepsis-an-ongoing-and-significant-challenge/be- ware-of-unusual-organisms-masquerading-as-skincontaminants

15 Berríos-Torres SI, Umscheid CA, Bratzler DW, et al; Healthcare Infection Control Practices Advisory Committee. Centers for Disease Control and Prevention Guideline for the prevention of surgical site infection, 2017. JAMA Surg 2017;152(08): 784-791

16 Beldame J, Lagrave B, Lievain L, Lefebvre B, Frebourg N, Dujardin F. Surgical glove bacterial contamination and perforation during total hip arthroplasty implantation: when gloves should be changed. Orthop Traumatol Surg Res 2012;98(04):432-440

17 Byrne AM, Morris S, McCarthy T, Quinlan W, O'byrne JM. Outcome following deep wound contamination in cemented arthroplasty. Int Orthop 2007;31(01):27-31

18 Ahn J-S, Lee H-J, Park E, Park I-Y, Lee JW. Suction drain tip culture after spine surgery: can it predict a surgical site infection? Asian Spine J 2015;9(06):863-868

19 Al-Maiyah M, Bajwa A, Mackenney P, et al. Glove perforation and contamination in primary total hip arthroplasty. J Bone Joint Surg Br 2005;87(04):556-559

20 Curcio D, Cane A, Fernández F, Correa J. Surgical site infection in elective clean and clean-contaminated surgeries in developing countries. Int J Infect Dis 2019;80:34-45

21 Bezstarosti H, Van Lieshout EMM, Voskamp LW, et al. Insights into treatment and outcome of fracture-related infection: a systematic literature review. Arch Orthop Trauma Surg 2019;139(01): 61-72

22 National Institute for Health and Care Excellence. Surgical site infections: prevention and treatment. LondonNational Institute for Health and Care Excellence (UK)2020. (NICE Guideline, No. 125.) Accessed December 23, 2021: https://www.ncbi.nlm.nih.gov/books/NBK542473/

23 Santoshi JA, Behera P, Nagar M, Sen R, Chatterjee A. Current surgical antibiotic prophylaxis practices: a survey of orthopaedic surgeons in India. Indian J Orthop 2020;55(03):749-757

24 Ahmad R, Tham J, Naqvi SGA, Butt U, Dixon J. Supports used for positioning of patients in hip arthroplasty: is there an infection risk? Ann R Coll Surg Engl 2011;93(02):130-132

25 Rajkumari N, Gupta AK, Mathur P, et al. Outcomes of surgical site infections in orthopedic trauma surgeries in a tertiary care centre in India. J Postgrad Med 2014;60(03):254-259

26 Khan MS, ur Rehman S, Ali MA, Sultan B, Sultan S. Infection in orthopedic implant surgery, its risk factors and outcome. J Ayub Med Coll Abbottabad 2008;20(01):23-25

27 Maksimović J, Marković-Denić L, Bumbasirević M, Marinković J, Vlajinac H. Surgical site infections in orthopedic patients: prospective cohort study. Croat Med J 2008;49(01):58-65

28 Uckay I, Kressmann B, Agostinho A, Landelle C, Al-Mayahi M, Pittet D. Anaerobes in clean orthopedic surgery? Is it a problem?. Antimicrob Resist Infect Control 2015;4:71 